\title{
Prediction of Group Learning Results from an Aggregation of Individual Understanding with Kit-Build Concept Map
}

\author{
Yusuke Hayashi ${ }^{(凶)}$, Toshihiro Nomura, and Tsukasa Hirashima \\ Graduate School of Advanced Science and Engineering, Hiroshima University, \\ Higashihiroshima, Japan \\ hayashi@lel.hiroshima-u.ac.jp
}

\begin{abstract}
With the development of information and communication technology, we can collect and analyze a variety of data for optimization. It is expected that the prediction of learning with the data enables a deep reflection for enhancing the learning experience. This paper describes a method to predict the group learning results from aggregation of an individual's understanding with the Kit-build concept map (KBmap). KBmap is a reconstruction-type concept map with automated diagnosis of the content. To test this method, we examined the prediction results from the data collected from a classroom lesson. The results show that most of the actual results are in good agreement with the prediction, and the comparison between the actual results and the predictions could be useful for the teacher.
\end{abstract}

Keywords: Concept map $\cdot$ Kit-build $\cdot$ Collaborative learning $\cdot$ Prediction

\section{Introduction}

In recent years, the forms of learning in schools have diversified, for example in the form of lectures, collaborative learning, and problem-based learning. In addition, with the evolving learning forms, the role of teachers in the classroom has changed from instructors who impart knowledge to the students to a facilitator for management of learning [1-3].

This study provides an environment for students to work collaboratively with concept maps [10] and for teachers to monitor their interactions. Network analysis analyzes the network structures, including actor-actor (social) network as well as actorartifact networks. Matsuzawa et al. [8] propose a tool for exploring the network structure of collaborative learning discourses.

Here, we propose a method to predict group learning results from the initial understanding of the members of the group based on a Kit-build concept map [6, 7, 11]. In this method, each student creates a concept map as their initial understanding of the subject. While learning as a group, students compare their concept maps and construct a kit-build concept map representing a consented understanding of what they have learned. The prediction shows the possibilities of the resulting concept maps from the understanding of students at the beginning of the group learning based on the patterns 
of individual understanding $[4,5,9]$. This paper also demonstrates an example of the prediction result of data from an actual lesson.

\section{Kit-Build Concept Map}

The kit-build concept map can provide a guideline for internalizing given information and a method to evaluate it. The kit-build concept map enables students to organize their understanding by reconstruction of the reference concept map called "goal map", and the teacher can assess students' concept maps by their automatic comparison with the reference concept map.

Figure 1 shows the goal map, and a kit made from the goal map in this study. Links and nodes connected to the central topic of the kit were not separated as the basic structure showed the viewpoints to create this map. The viewpoints represented by the connected nodes were "nature," "industry," and "culture." The task of the students for creating a map was to organize the instances of the viewpoints and to find intersections of the viewpoints.
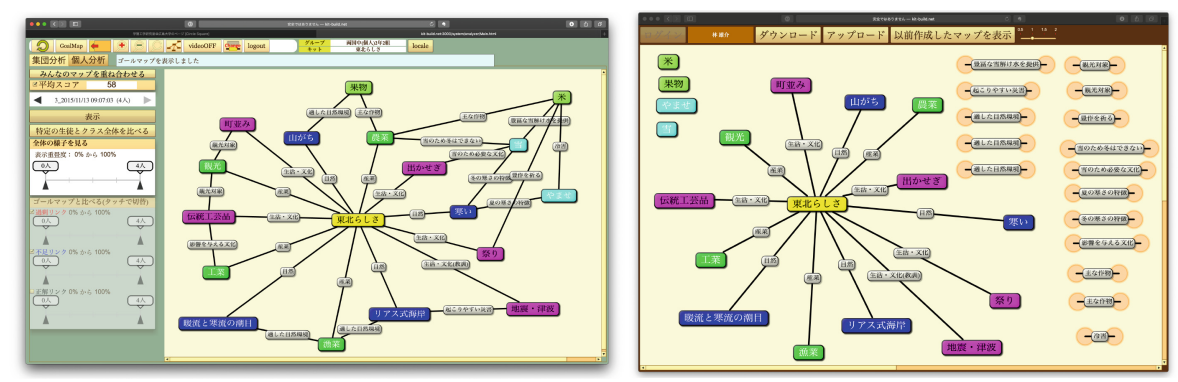

Fig. 1. The goal map and the kit made from the goal map

The KB map system [11] can automatically compare between the maps since the components of the goal map and students map are unified. The comparison may be performed for each proposition, and between students for each link, by comparing the goal map, to realize the group result prediction function. Proposition, which is determined from the above-described problems of group activities, becomes a goal map. Individual maps represent the understanding of each member of the group in the beginning of the learning process. The classification between the propositions, such as "the same proposition as the goal map (GM)," "different proposition from the GM," and "no opinion" is obtained by comparing the individual maps and the goal map. Then, it is possible to aggregate a combination thereof or matching rate for each group. 


\section{Prediction of the Group Learning Results}

Table 1 shows the classification of the proposition made by individuals in the group and pattern them. The propositions are categorized into three types based on the link between concepts: the same propositions as the reference map (the link connects the same concepts as the reference map), different propositions from the reference map (the link connects the different concepts from the reference map), and incomplete propositions (the link does not connect any concepts). For example, in pattern A, all the members have the same proposition as the reference concept map. By contrast, in patterns $\mathrm{D}-\mathrm{G}$, there is a conflict of propositions in the group.

Table 1. Proposition patterns in a group

\begin{tabular}{l|l|l|l}
\hline Pattern & Same as the GM & Different from the GM & No opinion \\
\hline A & Exist & Non-existent & Non-existent \\
\hline B & Non-existent & Exist & Non-existent \\
\hline C & Non-existent & Non-existent & Exist \\
\hline D & Exist & Exist & Non-existent \\
\hline E & Exist & Non-existent & Exist \\
\hline F & Non-existent & Exist & Exist \\
\hline G & Exist & Exist & Exist \\
\hline
\end{tabular}

We can anticipate the shared understanding of each proposition from the patterns of propositions in the group. The basic rules of prediction in this study are straightforward. If anyone in a group makes a proposition with a link, they select the proposition as their decision. However, if no one has any proposition with a link, they create a correct or wrong proposition or do not create any proposition. For example, in pattern A, their result is uniquely decided into the correct proposition. However, in pattern D, they can create a correct or incorrect proposition.

The proposed prediction calculates the maximum and the minimum result from the proposition patterns in the group. The maximum result occurs when the students in a group choose only correct propositions. The minimum result occurs when they choose some wrong propositions even if a single member in the group has opted for some correct propositions. In the patterns $\mathrm{D}$ or $\mathrm{G}$, the maximum result is derived when they choose the correct proposition, and the minimum score is derived when they choose the wrong proposition.

\section{Experimental Application to the Lesson Data}

This study used the data from a lesson where a total of 70 people from two classes in the second grade of junior high school participated. These lessons were taught as one lesson for each class. The topic was the Tohoku region of Japan in geography. The purpose of this lesson was to frame together the knowledge of nature, industry, 
traditions, and the culture of the Tohoku region as one structure and to make a common background for the discussion on their proposals for the reconstruction of the region in later lessons. Figure 2 shows the basic flow of the lesson.

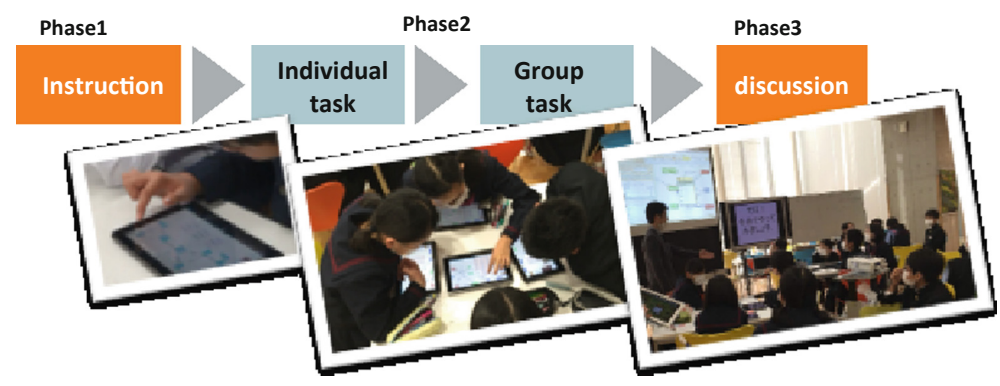

Fig. 2. The basic flow of the lesson.

In this study, we verified the accuracy of the prediction of the group task outcomes. The accuracy is measured by the range of the expected score and its validity. The range is the difference between the possible maximum and the minimum scores calculated from individual maps. The validity is whether the scores of the group maps fall into the predicted ranges.

Figure 3 shows the actual scores of the group results. The triangle indicates the actual group map score. In group A score prediction, the average prediction width was $23 \%$, and the predicted scores were approximately 20 points.

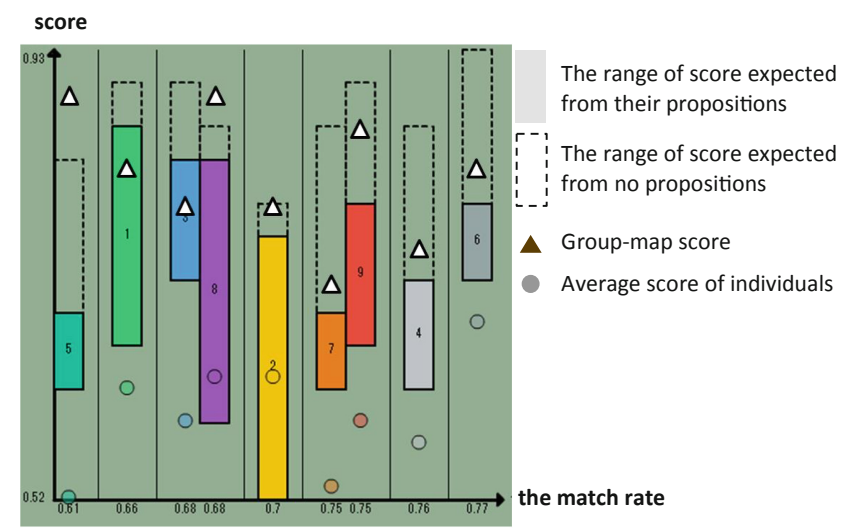

Fig. 3. Prediction of class A (sorted by the match rate) 


\section{Conclusion and Future Challenges}

In this case study, the score of the group activities had $20 \%$ width with more than $80 \%$ of the group scoring in the range of the predicted score as a result. About $80 \%$ of the proposition, even in the detailed analysis, has put the group results with the prediction rules. This shows the validity of the prediction method proposed in this study. In the questionnaire, the teacher pointed out the availability of the prediction graph. The group outcome prediction graph is expected to perform as the representation of the grasping ability of each group and the facilitation of group activities.

Future challenges are the verification of the use of the group outcome prediction function in the classroom by teachers and the learning effect of facilitation of group learning based on the prediction.

\section{References}

1. Carey, D.M.: Teacher roles and technology integration: moving from teacher as director to teacher as facilitator. Comput. Sch. 9(2), 105-118 (1994)

2. Dillenbourg, P., Patrick, J.: Technology for Classroom Orchestration. New Science of Learning, pp. 525-552. Springer, New York (2010). https://doi.org/10.1007/978-1-44195716-0_26

3. Grabinger, R.S., Dunlap, J.C.: Rich environments for active learning: a definition. Res. Learn. Technol. 3(2), 5-34 (1995)

4. Hayashi, Y., Nomura, T., Hirashima, T.: Orchestrating Individual- and group-learning in classroom with kit-build concept mapping. In: Proceedings of AIED2019, pp. 100-104 (2019)

5. Hayashi, Y., Nomura, T., Hirashima, T.: Propositional level analysis of collaborative learning with kit-build concept map. In: Proceedings of the 27th International Conference on Computers in Education (ICCE 2019), vol. 1, pp. 273-281 (2019)

6. Hirashima, T., Yamasaki, K., Fukuda, H., Funaoi, H.: Framework of kit-build concept map for automatic diagnosis and its preliminary use. Res. Pract. Technol. Enhanc. Learn. 10(1), 1-21 (2015)

7. Yamasaki, K., Fukuda, H., Hirashima, T., Funaoi, H.: Kit-build concept map and its preliminary evaluation. In: Proceedings of ICCE 2010, pp. 290-294 (2010)

8. Matsuzawa, Y., Oshima, J., Oshima, R., Niihara, Y., Sakai, S.: KBDeX: a platform for exploring discourse in collaborative learning. Procedia-Soc. Behav. Sci. 26, 198-207 (2011)

9. Nomura, T., Hayashi, Y., Suzuki, T., Hirashima, T.: Knowledge propagation in practical use of kit-build concept map system in classroom group work for knowledge sharing. In: Proceedings of ICCE 2014 Workshop, pp. 463-472 (2014)

10. Novak, J.D., Canas, A.J.: The theory underlying concept maps and how to construct them. Technical report IHMC CmapTools (2006)

11. Sugihara, K., et al.: Implementation of kit-build concept map with media tablet. In: Proceedings of WMUTE 2012, pp. 325-327 (2012) 